\title{
THE GENERALIZED DIFFERENTIAL TRANSFORM METHOD FOR SOLUTION OF A FREE VIBRATION LINEAR DIFFERENTIAL EQUATION WITH FRACTIONAL DERIVATIVE DAMPING
}

\author{
Deepanjan Das \\ Department of Mathematics, Ghani Khan Choudhury Institute of Engineering and Technology \\ Narayanpur, Malda, West Bengal-732141, India \\ deepanjan@gkciet.ac.in
}

Received: 16 November 2018; Accepted: 6 June 2019

\begin{abstract}
In the present paper, the Generalized Differential Transform Method (GDTM) is used for obtaining the approximate analytic solutions of a free vibration linear differential equation of a single-degree-of-freedom (SDOF) system with fractional derivative damping. The fractional derivatives are described in the Caputo sense.
\end{abstract}

MSC 2010: 26A33, 34A08, 35A22, 35R11, 35C10, $74 H 10$

Keywords: fractional differential equations, Caputo fractional derivative, generalized differential transform method, analytic solution

\section{Introduction}

Differential equations with fractional order are generalizations of classical differential equations of integer order and have recently been proved to be valuable tools in the modeling of many physical phenomena in various fields of science and engineering. By using fractional derivatives, a lot of work has been done for a better description of considered material properties. Based on enhanced rheological models, Mathematical modeling naturally leads to differential equations of fractional order and to the necessity of the formulation of the initial conditions to such equations. Recently, various analytical and numerical methods have been employed to solve linear and nonlinear fractional differential equations. The differential transform method (DTM) was proposed by Zhou [1] to solve linear and nonlinear initial value problems in electric circuit analysis. This method has been used for solving various types of equations by many authors [2-15]. DTM constructs an analytical solution in the form of a polynomial and different from the traditional higher order Taylor series method. For solving two-dimensional linear and nonlinear partial differential equations of fractional order, DTM is further developed as the 
Generalized Differential Transform Method (GDTM) by Momani, Odibat, and Erturk in their papers [16-18].

Recently, Vedat Suat Ertiirka and Shaher Momanib applied the generalized differential transform method to solve fractional integro differential equations [19]. The GDTM is implemented to derive the solution of space-time fractional telegraph equation by Mridula Garg, Pratibha Manohar and Shyam L. Kalla [20]. Manish Kumar Bansal, Rashmi Jain applied generalized differential transform method to solve the fractional order Riccati differential equation [21]. Aysegul Cetinkaya, Onur Kiymaz and Jale Camli applied generalized differential transform method to solve non linear PDE's of fractional order [22].

\section{Mathematical preliminaries on fractional calculus}

In the present analysis we introduce the following definitions [23, 24].

Definition 1. Let $\alpha \in R^{+}$On the usual Lebesgue space $L(a, b)$ integral operator $I^{\alpha}$ defined by

$$
\begin{aligned}
& I^{\alpha} f(x)=\frac{d^{-\alpha} f(x)}{d x^{-\alpha}}=\frac{1}{\Gamma(\alpha)} \int_{0}^{x}(x-t)^{\alpha-1} f(t) d t \text { and } \\
& I^{0} f(x)=f(x)
\end{aligned}
$$

is called Riemann-Liouville fractional integral operator of order $\alpha \geq 0$ and $a \leq x<b$.

It has the following properties:

I. $I^{\alpha} f(x)$ exists for any $x \in[a, b]$

II. $I^{\alpha} I^{\beta} f(x)=I^{\alpha+\beta} f(x)$

III. $I^{\alpha} I^{\beta} f(x)=I^{\beta} I^{\alpha} f(x)$

IV. $I^{\alpha} x^{\gamma}=\frac{\Gamma(\gamma+1)}{\Gamma(\alpha+\gamma+1)} x^{\alpha+\gamma}$

where $f(x) \in L[a, b], \alpha, \beta \geq 0, \gamma>-1$.

Definition 2. The Riemann-Liouville definition of fractional order derivative is

$$
{ }_{0}^{R L} D_{x}^{\alpha} f(x)=\frac{d^{n}}{d x^{n}}{ }_{0} I_{x}^{n-\alpha} f(x)=\frac{1}{\Gamma(n-\alpha)} \frac{d^{n}}{d x^{n}} \int_{0}^{x}(x-t)^{n-\alpha-1} f(t) d t,
$$

where $n$ is an integer that satisfies $n-1<\alpha<n$.

Definition 3. A modified fractional differential operator ${ }_{0}^{c} D_{x}^{\alpha}$ proposed by Caputo is given by

$$
{ }_{0}^{c} D_{x}^{\alpha} f(x)={ }_{0} I_{x}^{n-\alpha} \frac{d^{n}}{d x^{n}} f(x)=\frac{1}{\Gamma(n-\alpha)} \int_{0}^{x}(x-t)^{n-\alpha-1} f^{(n)}(t) d t,
$$


Where $\alpha\left(\alpha \in R^{+}\right)$is the order of operation and $n$ is an integer that satisfies $n-1<\alpha<n$.

It has the following two basic properties [25]:

I. If $f \in L_{\infty}(a, b)$ or $f \in C[a, b]$ and $\alpha>0$ then ${ }_{0}^{c} D_{x 0}^{\alpha} I_{x}^{\alpha} f(x)=f(x)$.

II. If $f \in C^{n}[a, b]$ and if $\alpha>0$ then ${ }_{0} I_{x}^{\alpha}{ }_{0}^{c} D_{x}^{\alpha} f(x)=f(x)-\sum_{k=0}^{n-1} \frac{f^{(k)}\left(0^{+}\right)}{k !} x^{k}$; $n-1<\alpha<n$.

Definition 4. For $m$ being the smallest integer that exceeds $\alpha$, the Caputo timefractional derivative operator of order $\alpha>0$, is defined as [26]

$$
\begin{aligned}
& D_{t}^{\alpha} u(x, t)=\frac{\partial^{\alpha} u(x, t)}{\partial t^{\alpha}} \\
& =\left\{\begin{array}{cc}
\frac{\partial^{m} u(x, \xi)}{\partial \xi^{m}} & ; \quad \alpha=m \in N \\
\frac{1}{\Gamma(m-\alpha)} \int_{0}^{t}(t-\xi)^{m-\alpha-1} \frac{\partial^{m} u(x, \xi)}{\partial \xi^{m}} d \xi & ; \quad m-1 \leq \alpha<m
\end{array}\right.
\end{aligned}
$$

Relation between Caputo derivative and Riemann-Liouville derivative:

$$
{ }_{0}^{c} D_{x}^{\alpha} f(x)={ }_{0}^{R L} D_{t}^{\alpha} f(x)-\sum_{k=0}^{m-1} \frac{f^{(k)}\left(0^{+}\right)}{\Gamma(k-\alpha+1)} x^{k-\alpha} ; m-1<\alpha<m
$$

Integrating by parts, we get the following formulae as given by [27]

I. $\int_{a}^{b} g(x){ }_{a}^{c} D_{x}^{\alpha} f(x) d x=\int_{a}^{b} f(x){ }_{x}^{R L} D_{b}^{\alpha} g(x) d x+\sum_{j=0}^{n-1}\left[{ }_{x}^{R L} D_{b}^{\alpha+j-n} g(x){ }_{x}^{R L} D_{b}^{n-j-1} f(x)\right]_{a}^{b}$

II. For $n=1, \int_{a}^{b} g(x){ }_{a}^{c} D_{x}^{\alpha} f(x) d x=\int_{a}^{b} f(x){ }_{x}^{R L} D_{b}^{\alpha} g(x) d x+\left[{ }_{x} I_{b}^{1-\alpha} g(x) \cdot f(x)\right]_{a}^{b}$

\section{Generalized one dimensional differential transform method}

Generalized differential transform of a function $y(x)$ in one variable is denoted by $Y_{\alpha}(k)$ and defined as follows [16-18]:

$$
Y_{\alpha}(k)=\frac{1}{\Gamma(\alpha k+1)}\left[\left(D_{x_{0}}^{\alpha}\right)^{k} y(x)\right]_{x=x_{0}}
$$

where $\alpha \in(0,1]$ and $\left(D_{x_{0}}^{\alpha}\right)^{k}=D_{x_{0}}^{\alpha}, D_{x_{0}}^{\alpha}, \ldots . ., D_{x_{0}}^{\alpha}$ (k-times). 
and the inverse generalized differential transform of $Y_{\alpha}(k)$ is given by

$$
y(x)=\sum_{k=0}^{\infty} Y_{\alpha}(k)\left(x-x_{0}\right)^{\alpha k}
$$

It has the following properties:

I. If $u(x)=v(x) \pm w(x)$ then $U_{\alpha}(k)=V_{\alpha}(k) \pm W_{\alpha}(k)$

II. If $u(x)=a v(x) ; a \in R$ then $U_{\alpha}(k)=a V_{\alpha}(k)$

III. If $U(x)=v(x) w(x)$ then $U_{\alpha}(x)=\sum_{r=0}^{k} V_{\alpha}(r) W_{\alpha}(k-r)$

IV. If $u(x)=\left(x-x_{0}\right)^{n \alpha}$ then $U_{\alpha}(k)=\delta(k-n)$

V. If $u(x)=D_{x_{0}}^{\alpha} v(x) ; 0<\alpha \leq 1$ then $U_{\alpha}(k)=\frac{\Gamma(\alpha(k+1)+1)}{\Gamma(\alpha k+1)} V_{\alpha}(k+1)$

VI. If $u(x)=x^{\lambda} f(x)$ where $\lambda>-1, f(x)$ has the generalized Taylor series expansion $f(x)=\sum_{n=0}^{\infty} a_{n}\left(x-x_{0}\right)^{n \alpha}$ and

a. $\beta<\lambda+1$ and $\alpha$ is arbitrary or

b. $\beta \geq \lambda+1, \alpha$ arbitrary and $a_{n}=0$ for $n=0,1,2, \ldots . m-1$, where $m-1<\beta \leq m$. Then (1) becomes

$$
U_{\alpha}(k)=\frac{1}{\Gamma(\alpha k+1)}\left[D_{x_{0}}^{\alpha k} u(x)\right]_{x_{0}}
$$

VII. If $u(x)=D_{x_{0}}^{\gamma} f(x), m-1<\gamma \leq m$ and the function $f(x)$ satisfies the conditions given in (VI) then $U_{\alpha}(k)=\frac{\Gamma(\alpha k+\gamma+1)}{\Gamma(\alpha k+1)} F_{\alpha}\left(k+\frac{\gamma}{\alpha}\right)$

where $U_{\alpha}(k), V_{\alpha}(k), W_{\alpha}(k)$ and $F_{\alpha}(k)$ are the differential transformations of the functions $u(x), v(x), w(x)$ and $f(x)$ respectively and $\delta(k-n)=\left\{\begin{array}{lll}1 & ; & k=n \\ 0 & ; & k \neq n\end{array}\right.$

\section{Solution of the free vibration linear differential equation of single-degree-of-freedom (SDOF) system with fractional derivative damping}

In this section, we consider the free vibration linear differential equation of single-degree-of-freedom (SDOF) system with fractional derivative damping 


$$
m \frac{d^{2} x(t)}{d t^{2}}+c \frac{d^{\alpha} x(t)}{d t^{\alpha}}+k x(t)=0
$$

subject to initial conditions $x(0)=p$ (constant) and $x^{\prime}(0)=q$ (constant),

where $\frac{d^{\alpha}}{d t^{\alpha}}$ is the fractional differential operator (Caputo derivative) of order $0<\alpha \leq 1$ and $m, c, k$ are the mass, damping and stiffness coefficient respectively.

Applying generalized one-dimensional differential transform (1) with $t_{0}=0$ on (3) we obtain

$$
X_{\alpha}(h)=-\frac{\Gamma\left(\alpha\left(h-\frac{2}{\alpha}\right)+1\right)}{m \Gamma\left(\alpha\left(h-\frac{2}{\alpha}\right)+3\right)}\left[c \frac{\Gamma\left(\alpha\left(h-\frac{2}{\alpha}+1\right)+1\right)}{\Gamma\left(\alpha\left(h-\frac{2}{\alpha}\right)+1\right)} X_{\alpha}\left(h-\frac{2}{\alpha}+1\right)+k X_{\alpha}\left(h-\frac{2}{\alpha}\right)\right]
$$

with $X_{\alpha}(0)=p$ and $X_{\alpha}\left(\frac{1}{\alpha}\right)=q$.

Taking $\alpha=1 / 2$, then (4) and (5) becomes

$$
X_{1 / 2}(h)=-\frac{\Gamma\left(\frac{1}{2} h-1\right)}{m \Gamma\left(\frac{1}{2} h+1\right)}\left[c \frac{\Gamma\left(\frac{1}{2} h-\frac{1}{2}\right)}{\Gamma\left(\frac{1}{2} h-1\right)} X_{1 / 2}(h-3)+k X_{1 / 2}(h-4)\right]
$$

with $X_{1 / 2}(0)=p$ and $X_{1 / 2}(2)=q$

Now utilizing the recurrence relation (6) and the initial condition (7), after a little simplification we obtain the following values of $X_{\frac{1}{2}}(k)$ for $k=0,1,2, \ldots \ldots$.

$$
\begin{aligned}
& X_{1 / 2}(1)=0 ; X_{1 / 2}(3)=0 ; X_{1 / 2}(4)=-\frac{k p}{m} \frac{1}{\Gamma(3)} ; X_{1 / 2}(5)=-\frac{c q}{m} \frac{1}{\Gamma(7 / 2)} ; \\
& X_{1 / 2}(6)=-\frac{k q}{m} \frac{1}{\Gamma(4)} ; X_{1 / 2}(7)=\frac{c k p}{m^{2}} \frac{1}{\Gamma(9 / 2)} ; X_{1 / 2}(8)=\frac{c^{2} q+k^{2} p}{m^{2}} \frac{1}{\Gamma(5)} ; \\
& X_{1 / 2}(9)=\frac{2 c k q}{m^{2}} \frac{1}{\Gamma(11 / 2)} ; X_{1 / 2}(10)=\left(\frac{k^{2} q}{m^{3}}-\frac{c^{2} k p}{m^{3}}\right) \frac{1}{\Gamma(6)} ;
\end{aligned}
$$




$$
\begin{aligned}
& X_{1 / 2}(11)=-\left(\frac{c\left(c^{2} q+k^{2} p\right)}{m^{3}}+\frac{c k^{2} p}{m^{3}}\right) \frac{1}{\Gamma(13 / 2)} \\
& X_{1 / 2}(12)=-\left(\frac{2 k q c^{2}}{m^{3}}+\frac{k\left(c^{2} q+k^{2} p\right)}{m^{3}}\right) \frac{1}{\Gamma(7)}
\end{aligned}
$$

and so on.

Now, from (2), we have

$$
x(t)=\sum_{h=0}^{\infty} X_{1 / 2}(h) t^{h / 2}
$$

Using the above values of $X_{1 / 2}(k) ; k=0,1,2, \ldots$. in (8) the solution of (3) is obtained as

$$
\begin{aligned}
& x(t)=p+q t-\frac{k p}{m} \frac{1}{\Gamma(3)} t^{2}-\frac{c q}{m} \frac{1}{\Gamma(7 / 2)} t^{5 / 2}-\frac{k q}{m} \frac{1}{\Gamma(4)} t^{3}+\frac{c k p}{m^{2}} \frac{1}{\Gamma(9 / 2)} t^{7 / 2} \\
& +\frac{c^{2} q+k^{2} p}{m^{2}} \frac{1}{\Gamma(5)} t^{4}+\frac{2 c k q}{m^{2}} \frac{1}{\Gamma(11 / 2)} t^{9 / 2}+\left(\frac{k^{2} q}{m^{3}}-\frac{c^{2} k p}{m^{3}}\right) \frac{1}{\Gamma(6)} t^{5} \\
& -\left(\frac{c\left(c^{2} q+k^{2} p\right)}{m^{3}}+\frac{c k^{2} p}{m^{3}}\right) \frac{1}{\Gamma(13 / 2)} t^{11 / 2}-\left(\frac{2 k q c^{2}}{m^{3}}+\frac{k\left(c^{2} q+k^{2} p\right)}{m^{3}}\right) \frac{1}{\Gamma(7)} t^{6}+\ldots .
\end{aligned}
$$

Taking $\alpha=1 / 3$, then (4) and (5) becomes

$$
X_{1 / 3}(h)=-\frac{\Gamma\left(\frac{1}{3} h-1\right)}{m \Gamma\left(\frac{1}{3} h+1\right)}\left[c \frac{\Gamma\left(\frac{1}{3} h-\frac{2}{3}\right)}{\Gamma\left(\frac{1}{3} h-1\right)} X_{1 / 3}(h-5)+k X_{1 / 3}(h-6)\right]
$$

with

$$
X_{1 / 3}(0)=p \text { and } X_{1 / 3}(3)=q
$$

Now utilizing the recurrence relation (10) and the initial condition (11), after a little simplification we obtain the following values of $X_{\frac{1}{3}}(k)$ for $k=0,1,2, \ldots \ldots$. 


$$
\begin{aligned}
& X_{1 / 3}(2)=0 ; X_{1 / 3}(1)=0 ; X_{1 / 3}(4)=0 ; X_{1 / 3}(5)=0 ; X_{1 / 3}(6)=-\frac{k p}{m} \frac{1}{\Gamma(3)} ; \\
& X_{1 / 3}(7)=0 ; X_{1 / 3}(8)=-\frac{c q}{m} \frac{1}{\Gamma(11 / 3)} ; X_{1 / 3}(9)=-\frac{k q}{m} \frac{1}{\Gamma(4)} ; X_{1 / 3}(10)=0 ; \\
& X_{1 / 3}(11)=\frac{c k p}{m^{2}} \frac{1}{\Gamma(14 / 3)} ; X_{1 / 3}(12)=\frac{k^{2} p}{m^{2}} \frac{1}{\Gamma(5)} ; X_{1 / 3}(13)=\frac{c^{2} q}{m^{2}} \frac{1}{\Gamma(16 / 3)} ; \\
& X_{1 / 3}(14)=\frac{2 c k q}{m^{2}} \frac{1}{\Gamma(17 / 3)} ; X_{1 / 3}(15)=\frac{k^{2} q}{m^{2}} \frac{1}{\Gamma(6)} ; X_{1 / 3}(16)=-\frac{c^{2} k p}{m^{3}} \frac{1}{\Gamma(19 / 3)}
\end{aligned}
$$

and so on.

Now, from (2), we have

$$
x(t)=\sum_{h=0}^{\infty} X_{1 / 3}(h) t^{h / 3}
$$

Using the above values of $X_{1 / 3}(k) ; k=0,1,2, \ldots$. in (12) the solution of (3) is obtained as

$$
\begin{aligned}
& x(t)=p+q t-\frac{k p}{m} \frac{1}{\Gamma(3)} t^{2}-\frac{c q}{m} \frac{1}{\Gamma(11 / 3)} t^{8 / 3}-\frac{k q}{m} \frac{1}{\Gamma(4)} t^{3}+\frac{c k p}{m^{2}} \frac{1}{\Gamma(14 / 3)} t^{11 / 3} \\
& +\frac{k^{2} p}{m^{2}} \frac{1}{\Gamma(5)} t^{4}+\frac{c^{2} q}{m^{2}} \frac{1}{\Gamma(16 / 3)} t^{13 / 3}+\frac{2 c k q}{m^{2}} \frac{1}{\Gamma(17 / 3)} t^{14 / 3}+\frac{k^{2} q}{m^{2}} \frac{1}{\Gamma(6)} t^{5} \\
& -\frac{c^{2} k p}{m^{3}} \frac{1}{\Gamma(19 / 3)} t^{16 / 3}+\ldots \ldots \ldots \ldots .
\end{aligned}
$$

Taking $\alpha=1 / 4$, then (4) and (5) becomes

$$
X_{1 / 4}(h)=-\frac{\Gamma\left(\frac{1}{4} h-1\right)}{m \Gamma\left(\frac{1}{4} h+1\right)}\left[c \frac{\Gamma\left(\frac{1}{4} h-\frac{3}{4}\right)}{\Gamma\left(\frac{1}{4} h-1\right)} X_{1 / 4}(h-7)+k X_{1 / 4}(h-8)\right]
$$

with $X_{1 / 4}(0)=p$ and $X_{1 / 4}(4)=q$

Now utilizing the recurrence relation (14) and the initial condition (15), after a little simplification we obtain the following values of $X_{1 / 4}(k)$ for $k=0,1,2, \ldots \ldots$ 


$$
\begin{aligned}
& X_{1 / 4}(2)=0 ; X_{1 / 4}(1)=0 ; X_{1 / 4}(3)=0 ; X_{1 / 4}(5)=0 ; X_{1 / 4}(6)=0 \\
& X_{1 / 4}(7)=0 ; X_{1 / 4}(8)=-\frac{k p}{m} \frac{1}{\Gamma(3)} ; X_{1 / 4}(9)=0 ; X_{1 / 4}(10)=0 ; \\
& X_{1 / 4}(11)=-\frac{c q}{m} \frac{1}{\Gamma(15 / 4)} ; X_{1 / 4}(12)=-\frac{k q}{m} \frac{1}{\Gamma(4)} ; X_{1 / 4}(13)=0 ; X_{1 / 4}(14)=0 ; \\
& X_{1 / 4}(15)=\frac{c k p}{m^{2}} \frac{1}{\Gamma(19 / 4)} ; X_{1 / 4}(16)=\frac{k^{2} p}{m^{2}} \frac{1}{\Gamma(5)} ; X_{1 / 4}(17)=0 ; \\
& X_{1 / 4}(18)=\frac{c^{2} q}{m^{2}} \frac{1}{\Gamma(22 / 4)} ; X_{1 / 4}(19)=\frac{2 c k q}{m^{2}} \frac{1}{\Gamma(23 / 4)} ; X_{1 / 4}(20)=\frac{k^{2} q}{m^{2}} \frac{1}{\Gamma(6)} \\
& X_{1 / 4}(21)=0 ; X_{1 / 4}(22)=-\frac{c^{2} k p}{m^{3}} \frac{1}{\Gamma(26 / 4)}
\end{aligned}
$$

and so on.

Now, from (2), we have

$$
x(t)=\sum_{h=0}^{\infty} X_{1 / 4}(h) t^{h / 4}
$$

Using the above values of $X_{1 / 4}(k) ; k=0,1,2, \ldots$. in (16) the solution of (3) is obtained as

$$
\begin{aligned}
& x(t)=p+q t-\frac{k p}{m} \frac{1}{\Gamma(3)} t^{2}-\frac{c q}{m} \frac{1}{\Gamma(15 / 4)} t^{11 / 4}-\frac{k q}{m} \frac{1}{\Gamma(4)} t^{3}+\frac{c k p}{m^{2}} \frac{1}{\Gamma(19 / 4)} t^{15 / 4} \\
& +\frac{k^{2} p}{m^{2}} \frac{1}{\Gamma(5)} t^{4}+\frac{c^{2} q}{m^{2}} \frac{1}{\Gamma(11 / 2)} t^{9 / 2}+\frac{2 c k q}{m^{2}} \frac{1}{\Gamma(23 / 4)} t^{19 / 4}+\frac{k^{2} q}{m^{2}} \frac{1}{\Gamma(6)} t^{5} \\
& -\frac{c^{2} k p}{m^{3}} \frac{1}{\Gamma(13 / 2)} t^{11 / 2}+\ldots \ldots
\end{aligned}
$$

Taking $\alpha=1 / 5$, then (4) and (5) becomes

$$
X_{1 / 5}(h)=-\frac{\Gamma\left(\frac{1}{5} h-1\right)}{m \Gamma\left(\frac{1}{5} h+1\right)}\left[c \frac{\Gamma\left(\frac{1}{5} h-\frac{4}{5}\right)}{\Gamma\left(\frac{1}{5} h-1\right)} X_{1 / 5}(h-9)+k X_{1 / 5}(h-10)\right]
$$


with $X_{1 / 5}(0)=p$ and $X_{1 / 5}(5)=q$

Now utilizing the recurrence relation (18) and the initial condition (19), after a little simplification we obtain the following values of $X_{1 / 5}(k)$ for $k=0,1,2, \ldots \ldots$

$$
\begin{aligned}
& X_{1 / 5}(2)=0 ; X_{1 / 5}(1)=0 ; X_{1 / 5}(3)=0 ; X_{1 / 5}(4)=0 ; X_{1 / 5}(6)=0 ; \\
& X_{1 / 5}(7)=0 ; X_{1 / 5}(8)=0 ; X_{1 / 5}(9)=0 ; X_{1 / 5}(10)=-\frac{k p}{m} \frac{1}{\Gamma(3)} ; X_{1 / 5}(11)=0 ; \\
& X_{1 / 5}(12)=0 ; X_{1 / 5}(13)=0 ; X_{1 / 5}(14)=-\frac{c q}{m} \frac{1}{\Gamma(19 / 5)} ; X_{1 / 5}(15)=-\frac{k q}{m} \frac{1}{\Gamma(4)} ; \\
& X_{1 / 5}(16)=0 ; X_{1 / 5}(17)=0 ; X_{1 / 5}(18)=0 ; X_{1 / 5}(20)=\frac{k^{2} p}{m^{2}} \frac{1}{\Gamma(5)} ; \\
& X_{1 / 5}(21)=0 ; X_{1 / 5}(22)=0 ; X_{1 / 5}(23)=\frac{c^{2} q}{m^{2}} \frac{1}{\Gamma(28 / 5)} ; \\
& X_{1 / 5}(24)=\frac{2 c k q}{m^{2}} \frac{1}{\Gamma(29 / 5)} ; X_{1 / 5}(25)=\frac{k^{2} q}{m^{2}} \frac{1}{\Gamma(6)} ; X_{1 / 5}(26)=0 ; \\
& X_{1 / 5}(27)=0 ; X_{1 / 5}(28)=-\frac{c^{2} k p}{m^{3}} \frac{1}{\Gamma(33 / 5)}
\end{aligned}
$$

and so on.

Now, from (2), we have

$$
x(t)=\sum_{h=0}^{\infty} X_{1 / 5}(h) t^{h / 5}
$$

Using the above values of $X_{1 / 5}(k) ; k=0,1,2, \ldots$. in (20) the solution of (3) is obtained as

$$
\begin{aligned}
& x(t)=p+q t-\frac{k p}{m} \frac{1}{\Gamma(3)} t^{2}-\frac{c q}{m} \frac{1}{\Gamma(19 / 5)} t^{14 / 5}-\frac{k q}{m} \frac{1}{\Gamma(4)} t^{3}+\frac{c k p}{m^{2}} \frac{1}{\Gamma(24 / 5)} t^{19 / 5} \\
& +\frac{k^{2} p}{m^{2}} \frac{1}{\Gamma(5)} t^{4}+\frac{c^{2} q}{m^{2}} \frac{1}{\Gamma(28 / 5)} t^{23 / 5}+\frac{2 c k q}{m^{2}} \frac{1}{\Gamma(29 / 5)} t^{24 / 5}+\frac{k^{2} q}{m^{2}} \frac{1}{\Gamma(6)} t^{5} \\
& -\frac{c^{2} k p}{m^{3}} \frac{1}{\Gamma(33 / 5)} t^{28 / 5}+\ldots \ldots \ldots \ldots . .
\end{aligned}
$$




\section{Conclusions}

In the present study, we have applied the Generalized Differential Transform Method (GDTM) to find the approximate analytic solution of the free vibration linear differential equation of single-degree-of-freedom (SDOF) system with fractional derivative damping. It may be concluded that the GDTM is a reliable technique to handle linear and nonlinear fractional differential equations. The GDTM provides more realistic series solutions compared with other approximate methods.

\section{References}

[1] Zhou, J.K. (1986). Differential Transformation and its Applications for Electrical Circuits. Wuhan: Huazhong University Press.

[2] Chen, C.K., \& Ho, S.H. (1999). Solving partial differential equations by two dimensional differential transform method. Appl. Math. Comput., 106, 171-179.

[3] Ayaz, F. (2004). Solutions of the systems of differential equations by differential transform method. Appl. Math. Comput., 147, 547-567.

[4] Abazari, R., \& Borhanifar, A. (2010). Numerical study of the solution of the Burgers and coupled Burgers equations by a differential transformation method. Comput. Math. Appl., 59, 2711-2722.

[5] Chen, C.K. (1999). Solving partial differantial equations by two dimensional differential transformation method. Appl. Math. Comput., 106, 171-179.

[6] Jang, M.J., \& Chen, C.K. (2001). Two-dimensional differential transformation method for partial differantial equations. Appl. Math. Comput., 121, 261-270.

[7] Kangalgil, F., \& Ayaz, F. (2009). Solitary wave solutions for the KDV and mKDV equations by differential transformation method. Choas Solitons Fractals, 41, 464-472.

[8] Arikoglu, A., \& Ozkol, I. (2006). Solution of difference equations by using differential transformation method. Appl. Math. Comput., 174, 1216-1228.

[9] Momani, S., Odibat, Z., \& Hashim, I. (2008). Algorithms for nonlinear fractional partial differential equations: A selection of numerical methods. Topol. Method Nonlinear Anal., 31, 211-226.

[10] Arikoglu, A., \& Ozkol, I. (2007). Solution of fractional differential equations by using differential transformation method. Chaos Solitons Fractals, 34, 1473-1481.

[11] Soltanalizadeh, B., \& Zarebnia, M. (2011). Numerical analysis of the linear and nonlinear Kuramoto-Sivashinsky equation by using Differential Transformation Method. Inter. J. Appl. Math. Mechanics, 7(12), 63-72.

[12] Tari, A., Rahimi, M.Y., Shahmoradb, S., \& Talati, F. (2009). Solving a class of twoDimensional linear and nonlinear Volterra integral equations by the differential transform method. J. Comput. Appl. Math., 228, 70-76.

[13] Nazari, D., \& Shahmorad, S. (2010). Application of the fractional differential transform method to fractional-order integro-differential equations with nonlocal boundary conditions. J. Comput. Appl. Math., 234 ,883-891.

[14] Borhanifar, A., \& Abazari, R. (2011). Exact solutions for non-linear Schr.dinger equations by differential transformation method. J. Appl. Math. Comput., 35, 37-51.

[15] Borhanifar, A., \& Abazari, R. (2010). Numerical study of nonlinear Schr.dinger and coupled Schr.dinger equations by differential transformation method. Optics Communications, 283, 2026-2031. 
[16] Momani, S., Odibat, Z., \& Erturk, V.S. (2007). Generalized differential transform method for solving a space- and time-fractional diffusion-wave equation. Physics Letters. A, 370(5-6), 379-387.

[17] Odibat, Z., \& Momani, S. (2008). A generalized differential transform method for linear partial Differential equations of fractional order". Applied Mathematics Letters, 21(2), 194-199.

[18] Odibat, Z., Momani, S., \& Erturk, V.S. (2008). Generalized differential transform method: application to differential equations of fractional order. Applied Mathematics and Computation, 197(2), 467-477.

[19] Ertiirka, V.S., \& Momanib, S. (2010). On the generalized differential transform method: application to fractional integro-differential equations. Studies in Nonlinear Sciences, 1(4), 118-126.

[20] Garg, M., Manohar, P., \& Kalla, S.L. (2011). Generalized differential transform method to Space-time fractional telegraph equation. Int. J. of Differential Equations, Hindawi Publishing Corporation, vol. 2011, article id. 548982, 9 pages, DOI: 10.1155/2011/548982.

[21] Bansal, M.K., \& Jain, R. (2015). Application of generalized differential transform method to fractional order Riccati differential equation and numerical results. Int. J. of Pure and Appl. Math., 99(3), 355-366.

[22] Cetinkaya, A., Kiymaz, O., \& Camli, J. (2011). Solution of non linear PDE's of fractional order with generalized differential transform method. Int. Mathematical Forum, 6(1), 39-47.

[23] Das. S. (2008). Functional Fractional Calculus, Springer.

[24] Miller, K.S., \& Ross, B. (1993). An Introduction to the Fractional Calculus and Fractional Diff. Equations. John Wiley and Son.

[25] Caputo, M. (1967). Linear models of dissipation whose q is almost frequency independent-ii. Geophys. J. R. Astron. Soc., 13, 529-539.

[26] Podlubny, L. (1999). Fractional differential equations: An introduction to fractional derivatives, fractional differential equations, to methods of their solution and some of their applications. Academic Press.

[27] Almeida, R., \& Torres, D.F. (2011). Necessary and sufficient conditions for the fractional calculus of variations with caputo derivatives. Communications in Nonlinear Science and Numerical Simulation, 16, 1490-1500. 